\title{
Hybrid tilted fiber grating based refractive index and liquid level sensing system
}

\author{
Zhijun Yan ${ }^{1,2}$, Chengbo Mou', Zhongyuan Sun${ }^{1}$, Kaimin Zhou ${ }^{1}$, Hushan Wang ${ }^{2}$, \\ Yishan Wang ${ }^{2}$, Wei Zhao ${ }^{2}$ and Lin Zhang ${ }^{1}$ \\ ${ }^{1}$ Aston Institute of Photonic Technologies, Aston University, Birmingham, UK, B4 \\ 7ET \\ ${ }^{2}$ State Key Laboratory of Transient Optics and Photonics, Xi' an Institute of Optics and \\ Precision Mechanics, Chinese Academy of Sciences, Xi'an 710119, China
}

Email: yanzhijun@gmail.com

\begin{abstract}
RI) and liquid level sensing system based on a hybrid grating structure comprising of a $45^{\circ}$ and an $81^{\circ}$ tilted fiber gratings (TFGs) that have been inscribed into a single mode fiber in series. In this structure, the $45^{\circ}$-TFG is used as a polarizer to filter out the transverse electric (TE) component and enable the $81^{\circ}$-TFG operating at single polarization for RI and level sensing. The experiment results show a lower temperature cross sensitivity, only about $7.33 \mathrm{pm} /{ }^{\circ} \mathrm{C}$, and a higher RI sensitivity, being around $180 \mathrm{~nm} / \mathrm{RIU}$ at $\mathrm{RI}=1.345$ and $926 \mathrm{~nm} / \mathrm{RIU}$ at $\mathrm{RI}=1.412$ region, which are significantly improved in comparison with long period fiber gratings. The hybrid grating structure has also been applied as a liquid level sensor, showing $3.06 \mathrm{~dB} / \mathrm{mm}$ linear peak ratio sensitivity.
\end{abstract}

Keyword: tilted fiber grating, polarization, refractive index sensor, liquid level sensor.

\section{Introduction}

Refractive index (RI) sensors have been widely applied in environment monitoring, bio-detection and food safety controlling areas [1-3]. Recently, optical fiber based RI sensors are attracting more interests due to their advantages of immunity to electromagnetic interference, compact size, high resolution, fast response and usage in harsh environment. A number of techniques have been explored to implement optical fiber based RI sensors, such as surface Plasmon resonance [4, 5], fiber based interferometer [6-8], fiber taper [9, 10], microfiber [11, 12] and fiber gratings [13, 14]. Among these, fiber gratings with intracore refractive index modification have various benefits over the other techniques. In a fiber grating based RI sensor, it can maintain the physical strength of a fiber device without modifying the fiber structure and format. Long period fiber gratings (LPGs), which can couple light from the fiber core mode to the cladding modes, are intrinsically sensitive to the surrounding medium RI [15]. It has been revealed that the RI sensitive region of $5^{\text {th }}$ order cladding mode of normal LPGs at $1500 \mathrm{~nm}$ lies predominantly within the range from $\mathrm{RI}=1.400$ to 1.444 with a nominal sensitivity of $1200 \mathrm{~nm} / \mathrm{RIU}$, and the typical RI sensitivity of LPGs is only around 90nm/RIU at the index sensing range of 1.33-1.400 [16]. Recently, by using LPGs of turning point or modifying structure, the RI sensitivity can be greatly improved in the aqueous solution $[17,18]$. However, LPGs still have high thermal cross-sensitivity, which affects the sensing accuracy for small RI changes. Tilted fiber gratings (TFGs) with small tilted structure have shown almost zero thermal cross-sensitivity, but their RI sensitivity is only $10 \mathrm{~nm} / \mathrm{RIU}$ [19]. Although it has been reported that the RI sensitivity can be enhanced to $555 \mathrm{~nm} / \mathrm{RIU}$ by small angle TFG based surface Plasmon resonance (SPR) sensor, complex nanomaterial manipulation process adds disadvantages [20]. Same to LPGs, an excessively tilted fiber gratings (ex-TFGs) can couple the forward propagating core mode to the high order forward propagating cladding

Manuscript received Month XX,XX .. 
modes, but have refractive index planes tilted at an angle larger than $70^{\circ}$, which may offer the combination of low thermal cross-sensitivity but high RI sensitivity [21]. Because of its asymmetric structure, Ex-TFGs have shown pronounced birefringence, which degenerates the normal cladding modes into two sets of modes with orthogonal polarization states, then showing dual-peak feature in the transmission spectrum. In our previous work, we have utilized ex-TFGs as twist, loading, liquid level and glucose sensors[22]. However in these experiments, a set of commercial polarizer and polarization controller was always used to ensure only one set of polarization peaks are selected for the measurement [23-26], which limits the size and operation of the sensor system. As reported in refs. [27, 28], a 45 ${ }^{\circ}$-TFG can be used as a TM mode pass filter, which can eliminate the TE resonances in the fiber. With the benefit of all fiber format, the $45^{\circ}$-TFG may be co-inscribed with the ex-TFG in the fiber to form a compact sensor structure and eliminate the use of the polarizer and polarization controller. In this work reported here, we proposed and demonstrated a fiber sensor based on a hybrid structure combining a $45^{\circ}$ - and an $81^{\circ}$-TFGs which were UV-inscribed in a single mode fiber in series. The fabricated hybrid grating was evaluated for RI and liquid level sensing.

\section{Theory and structure}

Same to an LPG, an ex-TFG can couple the forward-propagating core mode to the forward-propagating cladding modes but with high mode orders. Because the excessively tilted index fringes break the asymmetry of the fiber, the cladding modes coupled from core mode degenerated into two sets with orthogonal polarization states. The strongest light coupling of ex-TFG occurs at the wavelength determined by the phase matching condition [29]:

$\lambda=\left(n_{c o}^{\text {eff }}(\lambda)-n_{c l, m}^{i, e f f}(\lambda)\right) \frac{\Lambda_{G}}{\cos \theta} \quad \mathrm{i}=\mathrm{TE}$ or TM

Where $\lambda$ is the resonance wavelength, $n_{c o}^{\text {eff }}$ is the effective index of the core mode state, $n_{c l, m}^{i, e f f}$ is the effective index of $m^{\text {th }}$ cladding mode at the wavelength $\lambda ; \Lambda_{G}$ is the normal period of grating; $\theta$ is the tilt angle of the grating.

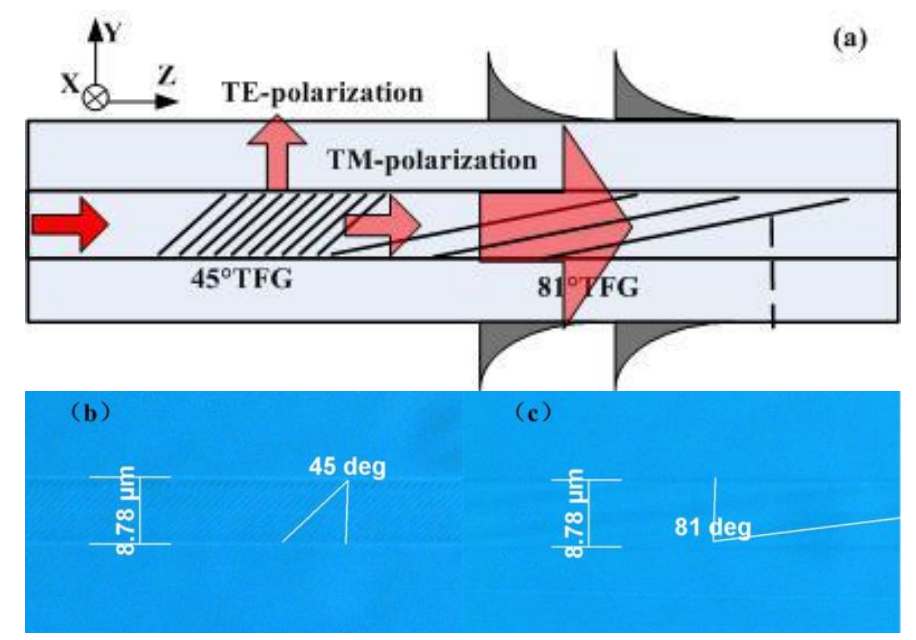

Fig. 1 (a) Schematic of a hybrid structure comprising a $45^{\circ}$ and an $81^{\circ}$ TFGs; the microscopy images of $45^{\circ}$-TFG (b) and $81^{\circ}$-TFG (c) in fiber core.

The schematic of the hybrid sensor structure is shown in Fig. 1(a). The TE component of the incident light passing through the $45^{\circ}$-TFG will be filtered out, and the remaining $\mathrm{TM}$ component will then propagate through the $81^{\circ}$-TFG. In a $45^{\circ}-\mathrm{TFG}$, the forward propagating core mode is coupled out of the fiber core by means of radiation modes, the $45^{\circ}$-TFG is therefore insensitive to the external temperature and RI , thus leaving the $81^{\circ}$-TFG performing RI or level sensing at single polarization. 
The general expression of environmental sensitivity of an ex-TFG may be written as [30]:

$$
\Delta \lambda=\gamma(\Gamma+\alpha) \lambda
$$

Where, $\gamma$ is the waveguide dispersion, $\Gamma$ is the environment dependence of waveguide dispersion and $\alpha$ is the material expansion caused by the changing of environment $(\alpha)$. The resonance peak of ex-TFG reported in this paper is the coupling to cladding mode at $24^{\text {th }}$ order, which is higher than the order of turning point cladding mode. According to ref. [30], the ex-TFGs have a negative value $\gamma$ factor, lower thermal dependence dispersion and higher RI dependence dispersion. In our experiment, we have proved that the ex-TFG is insensitive to temperature and highly sensitive to the surrounding medium RI.

\section{Sensor Fabrication}

The SMF-28 standard telecom single mode fiber used for TFG inscription was firstly hydrogen loaded at $80^{\circ} \mathrm{C}$ and 150 bar pressure for $48 \mathrm{~h}$ to increase its photosensitivity. To make sure that the $45^{\circ}$ and $81^{\circ}$ TFGs were inscribed along the same axis, the fiber orientation was marked before the UV-inscription. Due to the cylindrical shape of optical fiber, the UV fringes in the core are at a different angle as the external tilted angle [27]. To make sure the index fringes in the fiber core are at $45^{\circ}$ and $81^{\circ}$, the external inscription angles should be at $33.7^{\circ}$ and $76.5^{\circ}$, respectively. During the UV inscription, the $81^{\circ}$-TFG was firstly inscribed into the fiber by using an amplitude-mask with $6.6 \mu \mathrm{m}$ period and tilted at $76.5^{\circ}$ with respect to the fiber axis.
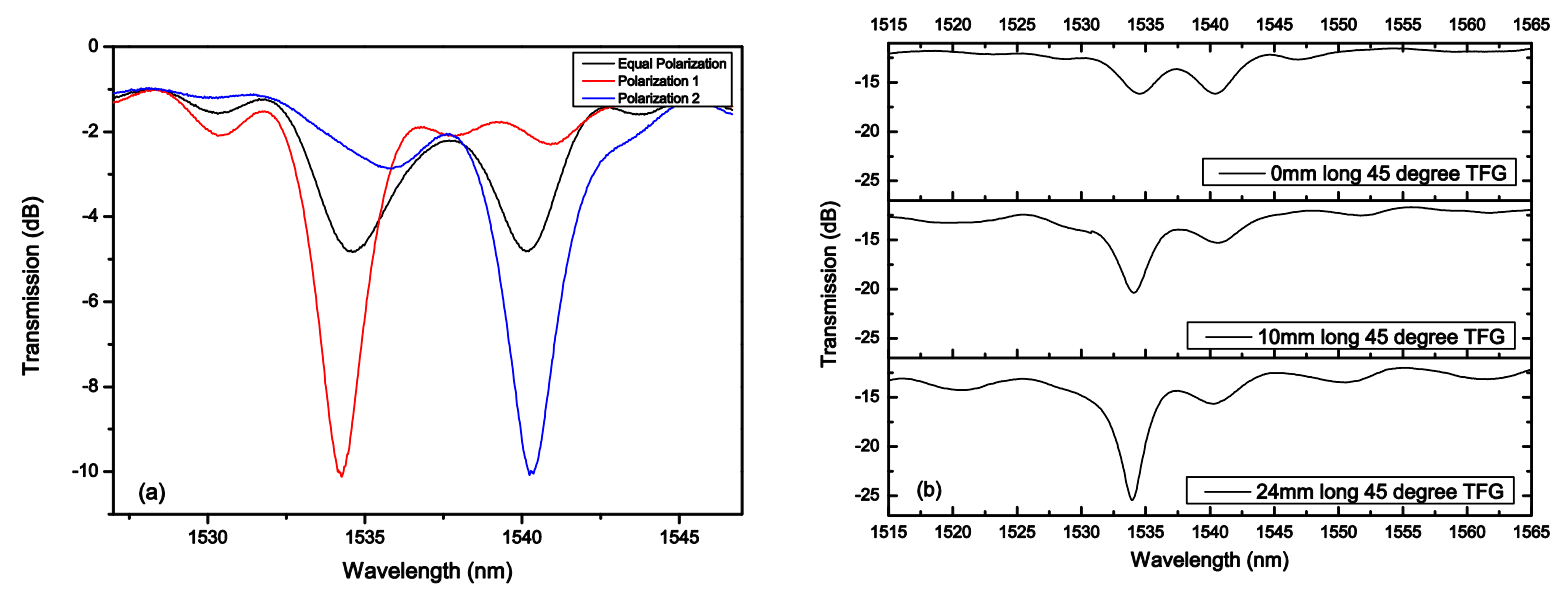

Fig. 2 Transmission spectra of (a) the single $81^{\circ}-\mathrm{TFG}$ at different polarizations and (b) the hybrid $45^{\circ}$-TFG and $81^{\circ}$-TFG when measured during the UV-exposure when the $45^{\circ}$-TFG reaching $0 \mathrm{~cm}, 10 \mathrm{~cm}$ and $24 \mathrm{~cm}$ length.

After the first UV-inscription, the $81^{\circ}$-TFG was measured by using a broad band source, a set of commercial polarizer and polarization controller (PC), and the experiment setup reported in refs. [23, 24]. The transmission spectra are shown in Fig. 2 (a). As seen from the figure, the spectra of $81^{\circ}$-TFG has two polarization dependent resonance peaks around $1534 \mathrm{~nm}$ and $1540.5 \mathrm{~nm}$, and by adjusting the PC, these two peaks can have equal strength (around $3 \mathrm{~dB}$ ), or switch to either polarization (TE or TM), e.g. one of the peaks disappears while the other grows into a strong attenuation peak of $\sim 10 \mathrm{~dB}$. The spectral separation between the paired peaks is around $6.5 \mathrm{~nm}$. Following the $81^{\circ}$-TFG inscription, a $24 \mathrm{~mm}$-long $45^{\circ}$-TFG structure was inscribed in front of the $81^{\circ}$-TFG in the same fiber by using a phase-mask with a period of $1800 \mathrm{~nm}$ and tilted angle at $33.7^{\circ}$. During the inscription of $45^{\circ}-\mathrm{TFG}$, the transmission of the hybrid structure was monitored. With increasing length of the $45^{\circ}$-TFG, the peak (TM mode) of the $81^{\circ}$-TFG at the shorter wavelength side was growing stronger, and the other one (TE 
mode) at longer wavelength became weaker. When the UV-inscription on the $24 \mathrm{~mm}$-long $45^{\circ}$-TFG completed, the strength of TM peak of the $81^{\circ}$-TFG reached $10 \mathrm{~dB}$ while the TE peak almost vanished (Fig. 2(b)). After the UV-inscription, the hybrid structure was examined under a $\times 100$ microscope objective (Axioscope-2 MOT plus (Zeiss), and we clearly observed the images of the $45^{\circ}$-TFG and $81^{\circ}$-TFG structures, as shown in Fig. 1 (b) and (c).

\section{Temperature and RI Sensing Experiments}

a. Temperature sensing

The hybrid structure of $45^{\circ}$-TFG and $81^{\circ}$-TFG structure was mounted on a temperature controlled peltier device for thermal response characterization, and the temperature was changed from $0^{\circ} \mathrm{C}$ to $60^{\circ} \mathrm{C}$ with $10^{\circ} \mathrm{C}$ increment. The thermal-induced wavelength shift of the TM peak of the hybrid structure is plotted in Fig. 3, which shows the thermal sensitivity is only about $7.33 \mathrm{pm} /{ }^{\circ} \mathrm{C}$. This thermal sensitivity is one order of magnitude lower than that of the normal LPGs and even less than the short period fiber Bragg gratings (FBGs). The low thermal cross-sensitivity is mainly attributed to the fact that high order forward propagating cladding modes have much lower thermal dependence of waveguide dispersion. As pointed out in ref [29], the thermal dependence of waveguide dispersion is approaching to zero at the higher cladding mode order [30].

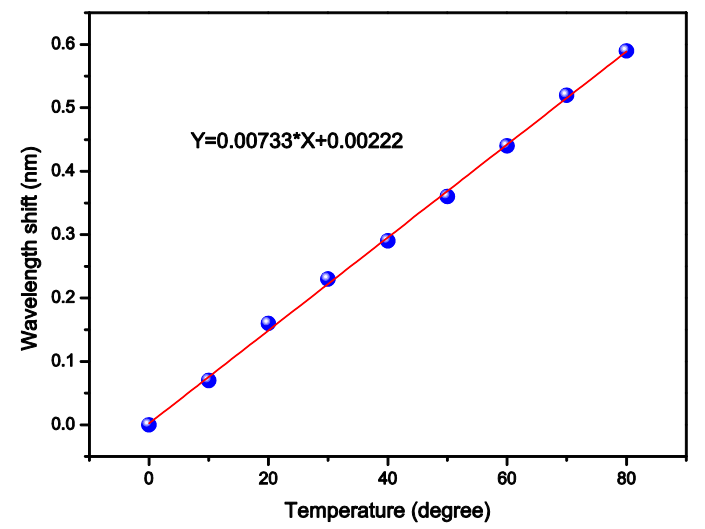

Fig. 3 Measured temperature sensitivity of the hybrid grating sensor concatenating a $45^{\circ}$-TFG and an $81^{\circ}$-TFG.

b. RI sensing

The RI sensing capability of the hybrid grating sensor based was evaluated by applying a series of index oils (from Cargille laboratory). In the experiment, to avoid the wavelength shift induced by the bending and axial strain, the sample was kept straight by clamping the grating on to the two translation stages with the same height. The index oil was placed on a flat glass substrate and the glass substrate was raised to immerge the grating fiber in the index oil. During the RI measurement process, the temperature was kept at a constant (around $25^{\circ} \mathrm{C}$ ). The index oils of 13 different indices ranging from 1.300 to 1.412 were applied to the hybrid grating in turn and the wavelength shift of the TM peak for each applied RI was recorded (the value of index oil was provided by Cargille lab, which was measured at 532nm). Note. after each RI measurement, the grating fiber was rinsed with methanol to remove the residual index oil on the surface of fiber till the original spectrum in air was restored on the optical spectrum analyzer. 


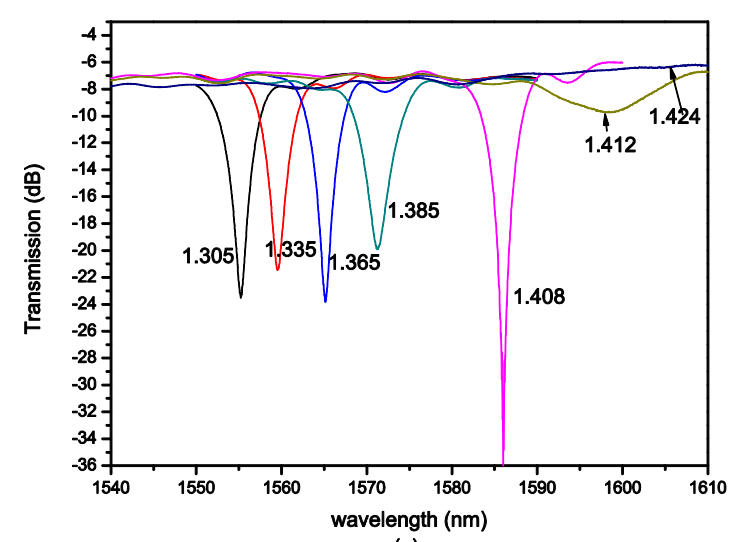

(a)

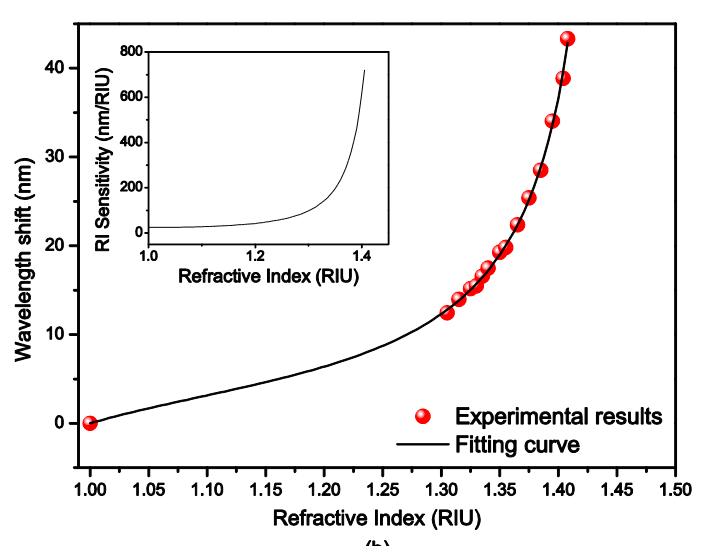

(b)

Fig. 5 (a) The transmission spectra of the hybrid $45^{\circ}-\mathrm{TFG}$ and $81^{\circ}-\mathrm{TFG}$ with different index oils applied to; (b) wavelength shift induced by different surrounding index oil: experimental results (•) and fitting curve (-), (inset: the RI sensitivity versus RI calculated from the fitting curve).

The shift of wavelength was almost instantaneously observed during the RI measurement. Fig.5(a) shows the transmission spectra of the grating sample with different index oils applied to it. As it shown in the figure, the single polarization resonant peak shifts to the longer wavelength with increasing RI and the peak has disappeared after the oil index value reached 1.412 , because this RI is larger than the effective index of the coupled high order cladding mode. We measured a total wavelength shift of $33.30 \mathrm{~nm}$ for the RI range from1.33 to1.412, which is 6 times higher than the LPGs reported in ref. [31]. The scattered dots plot in Fig. 5(b) shows the resonance wavelength as a function of RI for this hybrid grating structure. The plot exhibits a non-linear response, but perfectly fitted by the Nelder transition function (the smooth line curve), with a coefficient of determination of 0.9987 , very close to 1 . By differentiating the fitted curve, we retrieved a sensitivity curve about the RI (see the inset of Fig. 5b). From the results shown in the inset of Fig. 5(b), we can estimate that the RI sensitivities are around 926 $\mathrm{nm} / \mathrm{RIU}$ and $180 \mathrm{~nm} / \mathrm{RIU}$ at the RI of 1.412 and 1.345 , respectively. The typical RI sensitivity of LPGs is almost linear at the refractive index range between 1.33 and 1.40 , but is only around $60 \mathrm{~nm} / \mathrm{RIU}$, which is an order of magnitude lower compared to the hybrid TFG sensor [32]. Apart from achieving high RI sensitivity, these results clearly demonstrate that the hybrid sensor with a $45^{\circ}$-TFG can ensure single polarization RI sensing by the $81^{\circ}$-TFG, making the system simple and compact as no commercial polarization controller and polarizer are needed in the system.

\section{Application as a liquid level sensor}

Optical fiber grating based liquid level sensor have been attracting more interests for industrial applications. Comparing with traditional mechanical [33] and electrical [34] based liquid level sensor, the optical fiber grating based liquid level sensor is more suitable in conductive, flammable and explosive environments. In our previous work, we have reported an ex-TFG based liquid level sensor[26]. However, in the sensing experiment, a polarization controller and polarizer were also used to ensure only one peak excited in the transmission spectrum. The polarization state of light is very sensitive to operating status of sensors and will be affected by the effect such as bending, twisting, vibrating, and loading. The hybrid TFG structure will overcome polarization instability when used for liquid level sensing. We have used the same hybrid TFG sensor to evaluate its liquid level sensing capability by using a similar setup described in [26]. 


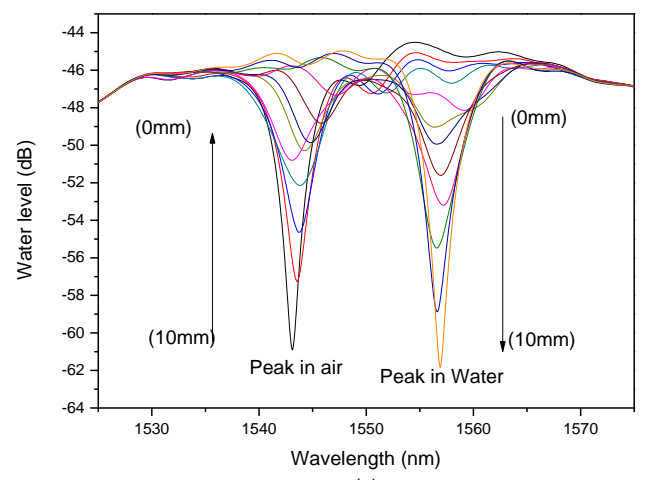

(a)

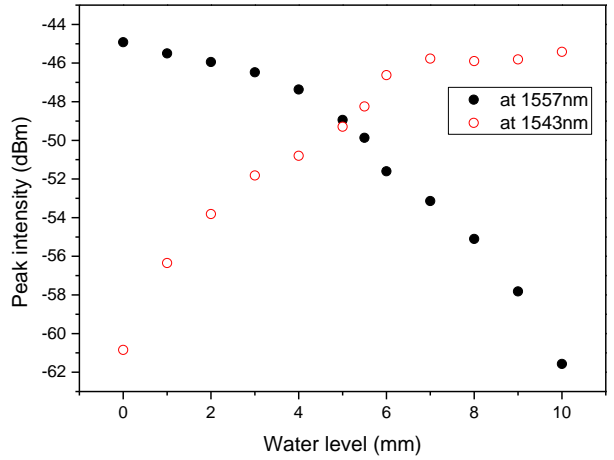

(b)

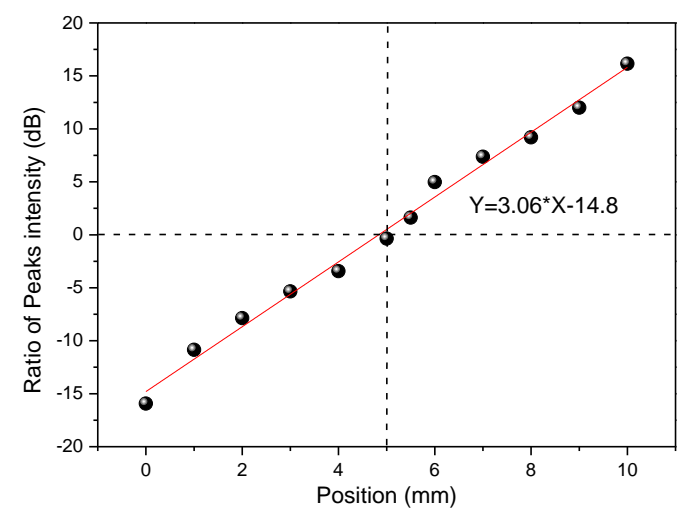

(c)

Fig. 6 (a) Transmission spectra of the hybrid $45^{\circ}$-TFG and $81^{\circ}-\mathrm{TFG}$ for TM resonance peaks surrounded by air and water; (b) the transmission intensity of the air-peak at $1543 \mathrm{~nm}$ and water-peak at $1557 \mathrm{~nm}$ measured with different water levels; (c) the intensity ratio of the air-peak and water-peak versus water level.

The length of $81^{\circ}$-TFG in the hybrid structure is around $10 \mathrm{~mm}$. The wavelength of TM resonance peak of $81^{\circ}$-TFG in air is at $1543 \mathrm{~nm}$ and shifted to $1557 \mathrm{~nm}$ when the grating is fully submerged in the water. In the experiment, we first set the whole $81^{\circ}$-TFG section exposed in air, and then increase the water level with the increment of $1 \mathrm{~mm}$ to gradually submerge the grating. As soon as part of the $81^{\circ}$-TFG immersing into the water, two resonance peaks generated: the one at $1543 \mathrm{~nm}$ corresponding to the part in air and the other at $1557 \mathrm{~nm}$ corresponding to the part in water. When the water level increasing, the air-peak strength decreases while the water-peak increases. After the whole grating section was immerged into the water, the peak at $1543 \mathrm{~nm}$ was completely disappeared and only the one at $1557 \mathrm{~nm}$ left in the spectrum. Fig. 6 (a) shows the spectral evolution of the hybrid grating for the process of the $81^{\circ}$-TFG wholly in air and totally covered into water. Fig. 6 (b) plots the transmission loss of the air-peak and water-peak, which showing similar results as reported in ref. [26]. A close examination shows that the air-peak at $1543 \mathrm{~nm}$ is not sensitive at the first $3 \mathrm{~mm}$ and the water-peak at $1557 \mathrm{~nm}$ is not sensitive at the last $3 \mathrm{~mm}$ water level position. Although the transmission loss against water level for both air- and water-peak is not linear (Fig. 6(b)), the re-plot of the transmission loss peak ratio (air-peak mines water-peak) against water level is linear, as clearly shown in Fig. 6(c). From Fig 6 (c), we can see that when the water level is at the middle of grating, the peak ratio is zero which means the air- and water-peak have the same transmission loss. The water level sensitivity can be estimated from Fig. 6(c) as $\sim 3.06 \mathrm{~dB} / \mathrm{mm}$. It should be pointed out that because the water level is encoded with the transmission loss, a 
low-cost intensity based interrogation method reported in ref [34] may be applied, instead using expensive optical spectrum analyser.

\section{Conclusions}

We have proposed and demonstrated a hybrid $45^{\circ}$-TFG and $81^{\circ}$-TFG sensor device UV-inscribed adjacently in a single mode fiber, in which the TE resonance peaks were totally eliminated. This hybrid grating structure has simplified the sensing system as it will not require the use of commercial polarization controller and polarizer. In the entire sensing measurement process, there was only a single TM peak to respond to the change of temperature, RI and water level. In comparison with an LPG, this hybrid structure showed a much lower thermal cross-sensitivity, as only $7.33 \mathrm{pm} /{ }^{\circ} \mathrm{C}$, and a much higher RI sensitivity in the RI region of 1.33-1.40. The RI sensitivity is exponentially increasing but reaches a cut-off value when the external RI exceeds the effective index of the high order cladding mode. The RI sensitivities are measured as $180 \mathrm{~nm} / \mathrm{RIU}$ and $926 \mathrm{~nm} / \mathrm{RIU}$ at $\mathrm{RI}=1.345$ and 1.404 regions, respectively. The hybrid grating device has also been evaluated for water level sensing showing a linear peak ratio response versus the water level with a sensitivity of $3.06 \mathrm{~dB} / \mathrm{mm}$.

\section{References}

[1]B. Lee, "Review of the present status of optical fiber sensors," Optical Fiber Technology, vol. 9, pp. 57-79, 2003.

[2]C. Xue, J. Wang, C. Liu, Y. Wang, D. Cui, and W. Zhang, "Research on optical biological sensor used as quantitative analysis of glucose," in Nano/Micro Engineered and Molecular Systems (NEMS), 2013 8th IEEE International Conference on, 2013, pp. 953-956.

[3]K. Narsaiah, S. Jha, R. Bhardwaj, R. Sharma, and R. Kumar, "Optical biosensors for food quality and safety assurance-a review," Journal of Food Science and Technology, vol. 49, pp. 383-406, 2012/08/01 2012.

[4]X. Wu, J. Zhang, J. Chen, C. Zhao, and Q. Gong, "Refractive index sensor based on surface-plasmon interference," Opt. Lett., vol. 34, pp. 392-394, 2009.

[5]P. K. Maharana, P. Padhy, and R. Jha, "On the Field Enhancement and Performance of an Ultra-Stable SPR Biosensor Based on Graphene," Photonics Technology Letters, IEEE, vol. 25, pp. 2156-2159, 2013.

[6]K. Zhou, Z. Yan, L. Zhang, and I. Bennion, "Refractometer based on fiber Bragg grating Fabry-Pérot cavity embedded with a narrow microchannel," Opt. Express, vol. 19, pp. 11769-11779, 2011.

[7]J. Tian, Y. Lu, Q. Zhang, and M. Han, "Microfluidic refractive index sensor based on an all-silica in-line Fabry\&\#x2013;Perot interferometer fabricated with microstructured fibers," Optics Express, vol. 21, pp. 6633-6639, 2013/03/11 2013.

[8]J. Wo, G. Wang, Y. Cui, Q. Sun, R. Liang, P. P. Shum, et al., "Refractive index sensor using microfiber-based MachZehnder interferometer," Optics Letters, vol. 37, pp. 67-69, 2012/01/01 2012.

[9]L. Cheng, Q. Sun-jie, C. Ye, X. Fei, and L. Yan-Qing, "Ultra-Sensitive Refractive Index Sensor With Slightly Tapered Photonic Crystal Fiber," Photonics Technology Letters, IEEE, vol. 24, pp. 1771-1774, 2012.

[10]P. Lu, L. Men, K. Sooley, and Q. Chen, "Tapered fiber MachZehnder interferometer for simultaneous measurement of refractive index and temperature," Applied Physics Letters, vol. 94, pp. 131110-131110-3, 2009.

[11]G. W. Jianghai Wo, Ying Cui, Qizhen Sun, Ruibing Liang, Perry Ping Shum, and Deming Liu, "Refractive index sensor using microfiber based Mach-Zehnder interferometer," Optics Letters, vol. 37, pp. 67-69, 2012. 
[12]J. Wen Bin, L. Huan Huan, T. Swee Chuan, C. Kin Kee, and A. Lim, "Ultrahigh Sensitivity Refractive Index Sensor Based on Optical Microfiber," Photonics Technology Letters, IEEE, vol. 24, pp. 1872-1874, 2012.

[13]S. Xuewen, Z. Lin, and I. Bennion, "Sensitivity characteristics of long-period fiber gratings," Lightwave Technology, Journal of, vol. 20, pp. 255-266, 2002.

[14]S. Li-Yang, A. P. Zhang, L. Wei-Sheng, F. Hong-Yan, and H. Sailing, "Optical Refractive-Index Sensor Based on Dual Fiber-Bragg Gratings Interposed With a Multimode-Fiber Taper," Photonics Technology Letters, IEEE, vol. 19, pp. 30-32, 2007.

[15]W. J. Stephen and P. T. Ralph, "Optical fibre long-period grating sensors: characteristics and application," Measurement Science and Technology, vol. 14, p. R49, 2003.

[16]V. Bhatia, "Applications of long-period gratings to single andmulti-parameter sensing," Opt. Express, vol. 4, pp. 457-466, 1999.

[17]X. Chen, K. Zhou, L. Zhang, and I. Bennion, "Dual-peak long-period fiber gratings with enhanced refractive index sensitivity by finely tailored mode dispersion that uses the light cladding etching technique," Appl. Opt., vol. 46, pp. 451-455, 2007.

[18]C. Kin Seng, L. Yunqi, N. Mei Nar, and D. Xiaoyi, "Analysis of etched long-period fibre grating and its response to external refractive index," Electronics Letters, vol. 36, pp. 966-967, 2000.

[19]C.-F. Chan, C. Chen, A. Jafari, A. Laronche, D. J. Thomson, and J. Albert, "Optical fiber refractometer using narrowband cladding-mode resonance shifts," Applied Optics, vol. 46, pp. 1142-1149, 2007/03/01 2007.

[20]C. C. Y. Shevchenko, M. A. Dakka, and J. Albert, "Polarization-selective grating excitation of plasmons in cylindrical optical fibers," Optics letters, vol. 35, pp. 637-639, 2010.

[21]K. Zhou, L. Zhang, X. Chen, and I. Bennion, "Optic sensors of high refractive-index responsivity and low thermal cross sensitivity that use fiber Bragg gratings of \&gt; $80^{\circ}$ tilted structures," Optics Letters, vol. 31, pp. 1193-1195, 2006/05/01 2006.

[22]Z. Y. Binbin Luo, Zhongyuan Sun, Jianfeng Li, and Lin Zhang, "Novel glucose sensor based on enzyme-immobilized $81^{\circ}$ tilted fiber grating," Opt. Express, vol. 22, pp. 30571-30578, 2014.

[23]K. Z. X. Chen, L. Zhang, and I. Bennion, "In-Fiber Twist Sensor Based on a Fiber Bragg Grating With 81 Tilted Structure," IEEE Photon. Technol. Lett., vol. 18, pp. 2596-2598, 2006.

[24]X. C. Rui Suo, Kaiming Zhou, Lin Zhang and Ian Bennion, "In-fibre directional transverse loading sensor based on excessively tilted fibre Bragg gratings " Measurement Science and Technology, vol. 20, p. 034015, 2009.

[25]Z. Y. Binbin Luo, Zhongyuan Sun, Jianfeng Li, and Lin Zhang, "Novel glucose sensor based on enzyme-immobilized $81^{\circ}$ tilted fiber grating," Optics Express, vol. 22, pp. 30571-30578, 2014.

[26]C. Mou, K. Zhou, Z. Yan, H. Fu, and L. Zhang, "Liquid level sensor based on an excessively tilted fibre grating," Optics Communications, vol. 305, pp. 271-275, 2013.

[27]Z. Yan, C. Mou, K. Zhou, X. Chen, and L. Zhang, "UV-Inscription, Polarization-Dependant Loss Characteristics and Applications of $45^{\circ}$ Tilted Fiber Gratings," J. Lightwave Technol., vol. 29, pp. 2715-2724, 2011.

[28]Z. Yan, K. Zhou, and L. Zhang, "In-fiber linear polarizer based on UV-inscribed $45^{\circ}$ tilted grating in polarization maintaining fiber," Opt. Lett., vol. 37, pp. 3819-3821, 2012.

[29]L. Z. Kaiming Zhou, Xianfeng Chen, and Ian Bennion, "Low Thermal Sensitivity Grating

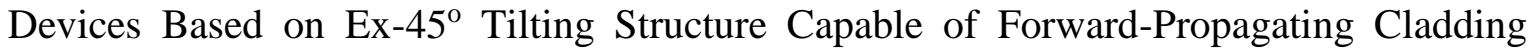
Modes Coupling," Journal of lightwave technology, vol. 24, pp. 5087-5094, 2006. 
[30]X. Shu, L. Zhang, and I. Bennion, "Sensitivity Characteristics of Long-Period Fiber Gratings," J. Lightwave Technol., vol. 20, p. 255, 2002.

[31]J. H. Chong, P. Shum, H. Haryono, A. Yohana, M. K. Rao, C. Lu, et al., "Measurements of refractive index sensitivity using long-period grating refractometer," Optics Communications, vol. 229, pp. 65-69, 2004.

[32]J.-L. Tang and J.-N. Wang, "Chemical Sensing Sensitivity of Long-Period Grating Sensor Enhanced by Colloidal Gold Nanoparticles," Sensors, vol. 8, pp. 171-184, 2008.

[33]B. W. Northway, N. H. Hancock, and T. Tran-Cong, "Liquid level sensors using thin walled cylinders vibrating in circumferential modes," Measurement Science and Technology, vol. 6, p. 85, 1995.

[34]F. N. Toth, G. C. M. Meijer, and M. vanderLee, "A new capacitive precision liquid-level sensor," in Precision Electromagnetic Measurements Digest, 1996 Conference on, 1996, pp. 356-357. 